\title{
La convivencia no es tan obvia
}

\author{
Alicia García Dalmás \\ (Universidad de la República, Uruguay)
}

Recibido: $7 / 3 / 2015$

Aprobado: 9/6/2015

\begin{abstract}
Resumen. La promoción de una mejor convivencia a través de la generación o mejora de espacios públicos, básicamente plazas y parques, sigue siendo una de las principales propuestas con las que las políticas públicas, por lo menos en Uruguay, buscan dar respuesta a lo que identifican como "problemas sociales acuciantes": la violencia, la inseguridad. El presente artículo toma como base una propuesta, la Estrategia por la Vida y la Convivencia, impulsada por el gobierno nacional que, entre otros aspectos, plantea que "el pacto de convivencia es un pacto de obviedad". Desde la comunicación propone aproximaciones y preguntas que buscan desnaturalizar lo obvio, profundizar en cómo se construyen vínculos y sentidos en relación a los territorios y la cotidianidad de quienes los habitan.
\end{abstract}

Palabras clave: comunicación / convivencia / cotidianidad / espacios públicos / políticas públicas

\section{Cohabitation Is Not so Obvious}

Summary. The promotion of a better coexistence across the generation or improvement of public spaces, namely squares and parks, continues to be one of the principal alternatives through with which public policies, at least in Uruguay, seek to respond to what they identify as "pressing social problems": violence, insecurity. This article takes as its foundation the Strategy for the Life and the Coexistence promoted by the national government that, among other aspects, states that "the pact of coexistence is a covenant of obviousness". From the field of communication, the article proposes approaches and questions that look to denaturalize the obvious, examine in depth how relationships and senses are constructed in relation to the territories and the daily lives of their inhabitants.

Key words: communication / coexistence / everyday life / public spaces / public policy 


\section{Puntos de partida}

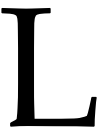

a Estrategia por la Vida y la Convivencia (EVC), planteada en 2012 e impulsada por el presidente uruguayo José Mujica, explicita desde el gobierno nacional la búsqueda de un nuevo posicionamiento del tema (buscando descentrarlo de la inseguridad). Propone un marco explicativo, propuestas y "medidas", algunas a través de normas y disposiciones para ser tratadas en el Parlamento, otras a través de un diálogo con la sociedad civil. Las acciones territorializadas, las que se refieren a las personas en tanto habitantes de una zona, de un barrio, son el marco de nuestro trabajo.

Partiendo de la idea de que socialmente hay "pérdidas", de la existencia de aspiraciones compartidas por "mayorías", de pautas de convivencia comunes, de valores y actitudes socialmente validados, de acuerdos, la Estrategia plantea que "el pacto de convivencia es un pacto de obviedad". Desde un abordaje comunicacional lo que buscamos es justamente aportar miradas que busquen una desnaturalización, entendida como ruptura de lo obvio, un abordaje crítico que permita profundizar en los sentidos, las modalidades de reproducción, producción, creación cotidiana del "mundo" y las relaciones, de lo privado y lo público, que no solo complejice las explicaciones sino que aporte a la construcción de alternativas.

Montevideo, el área metropolitana, el país, se transforma en escenario don- de como actores estamos involucrados en una "historia" que vemos, que oímos, que nos dice cosas, que cuenta sucesos acerca de nosotros, pero que nosotros a su vez contamos a otros sobre nuestra cotidianidad, nuestros miedos, nuestros sueños, nuestras esperanzas. Historias que se cuentan en el almacén, el supermercado o la feria, en la plaza, en la calle o en el ómnibus, en la escuela o el liceo, en el lugar de trabajo, en el boliche, en el estadio, en la radio, en la tele o en las redes sociales. Que hablan de lo que pasó a la vuelta de la esquina, pero también en el mundo, sobre lo de ayer y lo de hace muchos años, sobre lo que tenemos en común y sobre lo que nos diferencia. Tiene que ver con los medios, pero también con los territorios, como escenarios de comunicación donde se construyen y circulan cotidianamente saberes y sentidos.

Este trabajo es una aproximación preliminar con base en documentos de definición de políticas y estrategias, de elementos surgidos de anteriores procesos de investigación, de primeros acercamientos a un territorio. La investigación busca acercarse a nuevos escenarios y narraciones para pensar la sociedad como coexistencia o como unidad en la diversidad (Castoriadis, 1989), acceder a los conocimientos cruciales, los nudos o puntos estratégicos (Morin, 1999), buscar "pautas que conecten" (Bateson, 1997), preguntas verdaderas que incluyan la duda y la incertidumbre y que permitan aprehender la "libertad montaraz de las 
prácticas" (Certeau, 2000), recreación y construcción de vínculos y sentidos en el marco de tramas complejas, resistiendo a la tentación de relatos únicos y clausurados.

\section{Algo anda mal. ¿Seguridad o convivencia?}

Desde la percepción que miden algunas de las encuestadoras de opinión en Uruguay, la "seguridad pública" ha pasado a ser el "principal problema". Así, por ejemplo, ante la pregunta de la encuesta nacional que lleva adelante Factum ${ }^{1}$, una de las empresas de medición de "opinión pública", - ¿cuál de estos es a su juicio el principal problema que existe actualmente en el Uruguay?-, un $42 \%$ de los consultados planteó la seguridad pública, un $15 \%$ la educación, un $13 \%$ drogas y un $11 \%$ la violencia doméstica. La pobreza, por ejemplo, figura con un $5 \%$ y el trabajo/ empleo/desempleo con un $3 \%$.

El sociólogo Rafael Paternain (2 de febrero de 2011), docente universitario y responsable del observatorio de criminalidad del Ministerio del Interior hasta 2010, plantea que el sentimiento de inseguridad es una construcción colectiva y una vivencia cotidiana que tiene raíces y motivos más hondos que la mera evolución del delito. Hace lo que la gente cree, vive, siente y piensa sobre la "calidad de la convivencia". Los medios masivos de comunicación se identifican claramente como amplificadores de un sentir que se expresa en "esto no da para más", en la sensación de que nos puede suceder a todos y en cualquier momento. Muchos de los discursos se centran además en "los menores" como principales responsables de la inseguridad.

Frente a estas percepciones sobre la (in)seguridad y algunos hechos específicos de violencia que conmovieron a la sociedad $^{2}$, en junio de 2012 el Gabinete de Seguridad -integrado por los ministerios del Interior, Desarrollo Social (Mides) y Defensa Nacional- presentó oficialmente el documento Estrategia por la Vida y la Convivencia (EVC). Los puntos de partida del documento son que hay una "pérdida de respeto por la vida humana y la convivencia ciudadana, y ese es el corazón del problema que la sociedad uruguaya toda (Estado y

1 Ficha técnica: sondeo permanente multimétodo, entrevista en hogares del $100 \%$ del electorado (incluyendo zonas rurales, periféricas, asentamientos), de 15 y más años. Muestra de hogares, por conglomerado, polietápica, total de casos 968, realizada en el cuarto trimestre de 2012, ponderación por lema en elecciones nacionales de 2009 y por tres macrogeneraciones electorales, disponible en $\mathrm{http}: / / \mathrm{ww} w . f a c t u m . e d u . u y / n o d e / 855$

2 En una diversidad que fue desde situaciones de violencia en el deporte a asesinatos supuestamente cometidos por enfermeros hacia enfermos terminales en centros de salud públicos y privados de Montevideo. 
ciudadanos) debemos enfrentar" (EVC, p. 1). Si la inseguridad pública es el problema, la convivencia es la solución y el objetivo es vencer el miedo "a partir de garantizar los derechos humanos y la seguridad de sus habitantes" (EVC, p. 2). Una convivencia a la que aspiraría "la enorme mayoría de los uruguayos" que enfrenta una "variada gama de situaciones de violencia" (EVC, p. 4) y que se define como "ejercicio de convivir y vivir en compañía de otros", que requiere "apego a normas básicas de comportamiento y respeto a la ley" (EVC, p. 5). Se dice que las pautas de convivencia "son parte de un conjunto de valores y actitudes que socialmente están validadas y funcionan como un acuerdo. En definitiva 'el pacto de convivencia es un pacto de obviedad' y podemos y debemos potenciar valores alternativos" (EVC, p. 4).

Los discursos y acciones en relación a la convivencia y el espacio público, tanto desde el Estado, desde los vecinos organizados a nivel territorial, en diversos medios y plataformas, parten de que algo está "mal" o no está como se espera que esté.

En el marco de la crisis de 2002 realizamos un proceso de investigación participativa en una zona de
Montevideo $^{3}$, ciudad capital, donde aparecía la referencia a una "época de oro", un pasado de bienestar marcado desde el trabajo, la comida, los espacios de esparcimiento y modalidades vinculares o de relacionamiento, las organizaciones de todo tipo. En un contexto de crisis y por tanto de deterioro general, aparecía ya la preocupación por la seguridad. Las personas con quienes realizamos la investigación sentían un cambio importante. Decían, por ejemplo, que los robos "no existían" antes, el barrio era precioso, se dejaba la puerta sin llave o los animales domésticos sueltos en las calles. Frente a esa imagen idealizada, se identificaba un proceso de "enrejamiento", de "sálvese quien pueda", sentimientos de inseguridad. "Unos" que rompen, roban, piden limosna, reciben asistencia, no valoran ciertas cosas. "Miedo" de unos a otros. Sin embargo, al profundizar aparecían referencias a peleas y enfrentamientos en eventos deportivos, castigos de padres a hijos, represión policial a sindicalistas desde la década de 1950. Sin embargo, cuando se contrastaba con el presente, se decía que aquello era otra cosa, "más sano", "con valores".

3 La investigación se titulaba "Reconfiguración de espacios organizativos y participación ciudadana", realizada en el marco de una convocatoria abierta de la Comisión Sectorial de Investigación Científica (CSIC) de la Universidad de la República a proyectos de investigación que atendieran la situación de emergencia social en el año 2003. Se desarrolló en la zona 14 de Montevideo (actual Municipio A), situado en la zona oeste del Departamento. 
Por esa época, en la misma zona, realizamos una actividad ${ }^{4}$ donde se consultó a dos mil adolescentes: quienes concurrían a centros educativos, los que se reunían en la Plaza de Deportes, iban a los Centros Juveniles y a otras instituciones y grupos de la zona. Se les plantearon tres preguntas en un formulario: ¿Qué te gustaría cambiar?, ¿Por qué crees que las cosas no cambian?, ¿Y vos, qué podés hacer? Entre las respuestas destacaban las referidas a las vivencias en relación al "caos" (la crisis) y "el mundo de maldad en que vivimos". La violencia, los robos, los ladrones, la falta de rigurosidad en la justicia, los asesinatos, los accidentes, las drogas, los atentados, la guerra, el gobierno, la contaminación por plomo, la ignorancia, las injusticias, la gente que vive en la calle, los que no tienen para comer, los malos tratos en la familia, la mala gente. Situaciones y personas se entrelazaban en un mundo que anda "mal", abarcando ámbitos que iban desde lo familiar, lo vecinal, hasta lo mundial (como la guerra y los atentados), desde quienes no pagan los impuestos a quienes les pegan a sus hijos.

Si partimos de que cada sociedad no se reconoce a sí misma como creación, se piensa y se nombra como "la" sociedad, cuando ocurre un incidente y peligra el compromiso, es la misma realidad la que está en peligro, cualquier desviación de la realidad del orden institucional aparece como una desviación de la realidad (Berger y Luckmann, 2001).

\section{Lo común, lo diferente, lo desigual}

En la Estrategia por la Vida y la Convivencia se habla de un "fenómeno multidimensional", identificando como causas procesos de exclusión, de segmentación educativa y residencial, también se incluye la búsqueda del "éxito individual", niveles de consumo, "meteóricas carreras", como expresión de una "escala de valores distorsionada" (EVC, p. 3) promovida por los medios masivos, un consumismo que "altera la propia convivencia ciudadana y mercantilizan el concepto de felicidad" (EVC, p. 3). Se mezcla así en esta "multidimensionalidad" una diversidad de temáticas que aparecen como "lo que es", lo dado.

Más allá de los procesos de crecimiento y mejora del contexto macroeconómico en la región después de la crisis de los años 2000, de las políticas sociales de inclusión, persiste la pobreza estructural intergeneracional, el desenganche con el mundo educativo

4 Hacete Oír fue una propuesta llevada adelante por la Asociación Civil El Tejano, que también desarrolla sus actividades en el Municipio A de Montevideo. La primera edición se realizó entre 2000 y 2001. Incluía una etapa de consulta y espacios de propuesta con adolescentes y jóvenes. 
y del trabajo. En Uruguay, si bien se da una mejora en indicadores de pobreza (12,4 \% de la población en 2012), en el coeficiente de Gini, que bajó de 0,404 en 2011 a 0,379 en 2012, la pobreza sigue afectando mayoritariamente a generaciones jóvenes, en particular niños en edad escolar.

La desigualdad persiste y tiene dimensión económica, territorial, etárea. Territorialmente se localiza en zonas del país y barrios de la ciudad. En relación a la edad, afecta más a niños, niñas, adolescentes y jóvenes. Las sucesivas desestabilizaciones profundizaron procesos donde la dignidad personal fue avasallada. El otro aparece más como "carente" (o como amenazante) y no como ciudadano, profundizando los procesos de estigmatización y victimización. Cuando los demás son una amenaza, se tiende a encerrarse en el espacio íntimo, se renueva el discurso criminalizador, la vida de algunos sujetos deja de tener importancia. Se da una dislocación entre ciudadanía y solidaridad-responsabilidad.

La crisis de la "igualdad" como fundamento de lo común, el crecimiento de las desigualdades como indicio y motor de descomposición de lazos sociales y solidaridad (Rosanvallon, 2012), no solo tiene que ver con la violencia sino también con la tolerancia, una "pasividad práctica". Una impotencia donde el mayor conocimiento no lleva a la transformación sino a la búsqueda de chivos emisarios y el refugio en pensamientos mágicos. La "reducción de desigualdades" no tiene clara cuál es la imagen positiva de lo deseable. La distribución de servicios y beneficios pasa a ocupar el lugar de los derechos y la ciudadanía. Cuesta instalar en la producción y la reproducción una cultura de valores que priorice los derechos humanos y las responsabilidades universales (Coraggio, 2007).

\section{¿Quién propone qué? ¿Quién se hace cargo de qué?}

La Estrategia por la Vida y la Convivencia plantea "construir comunidad en los territorios" como pertenencia a un proyecto común, a valores compartidos, recuperar lugares para "estar", "vivir", "intercambiar". Lugares de tránsito, intercambio, generadores de encuentros. La superación del temor se plantea con la recuperación de espacios públicos, una "acupuntura urbana", una "nueva piel" de la ciudad, convocadora a la "caricia" y no a la "distancia". "Lo mejor para quienes más lo necesitan para poder saldar una deuda histórica y garantizar la viabilidad del país como proyecto de vida común" (EVC, p. 6).

Para la implementación se identificaron siete zonas como prioritarias, seleccionadas tomando como base el censo del 2011 del Instituto Nacional de Estadística y el relevamiento realizado por el Ministerio de Desarrollo Social en 2011 y 2012. Las intervenciones se plantean como puntuales (un año y medio) con inversión en 
infraestructura social y equipamiento, profundización en el acceso a servicios sociales básicos y estrategias de capacitación para jóvenes y mujeres jefas de hogar. Buscan fortalecer la presencia del Estado en estos barrios, con una batería importante de acciones, dejando servicios públicos instalados y articulando lo ya existente.

La propuesta base fue elaborada por el sociólogo y consultor Gustavo Leal ("Más barrio, más vida"). Se plantea como una estrategia "de inclusión social territorializada en los sectores de extrema pobreza del área metropolitana del país", en el marco del "urbanismo social", generando escenarios "dignos" que dinamicen la transformación social.

Más barrio porque el objetivo es fortalecer la trama urbana, mejorar la convivencia y el contacto cara a cara. Busca recuperar la habitabilidad y las relaciones de cercanía entre las personas de las comunidades. Más vida porque el centro del conjunto de las acciones apunta a elevar la calidad de vida de las poblaciones seleccionadas, fortaleciendo la autoestima y el sentido de pertenencia a la sociedad. Más vida es pues más ciudadanía efectiva (Leal, s. f., p. 1).

Plantea intervenciones "sustantivas" y "contundentes" en áreas geográficas donde se concentraría la mayoría de familias indigentes y vulnerables. Las acciones iniciadas serían luego gestionadas por vecinos, para lo cual se fortalecerían sus organizaciones, además de "voluntarios" (Programa de
Movilización Social de Voluntarios Solidarios) que combinarían acciones con los ministerios.

La crisis de los años noventa planteó un nuevo rol de las organizaciones de la sociedad civil. De ser un sector ligado a organizaciones más "tradicionales" (como por ejemplo los sindicatos) pasaron a tener una mayor diversificación y diversidad. En el marco de una reducción del rol del Estado se pasó a la autorresolución de demandas, a acciones colectivas en relación a hechos puntuales donde las llamadas ONG de promoción y desarrollo pasaron a tener otro papel, pero que también fue cambiando el rol de las organizaciones más "barriales", vecinales.

En un universo amplio y heterogéneo de proyectos, de estrategias de intervención, de formas de movilización y protesta, se dio una transferencia desde el Estado de parte de sus funciones, pero reduciendo la capacidad de incidencia a ámbitos focalizados y poco articulados. Al tener su sustentabilidad económica ligada al Estado (o a los organismos internacionales), les generó vulnerabilidad a las "modas" y exigencias de sus "financiadores". Se produjo un cambio y heterogeneidad de la demanda, con una conflictividad centrada no tanto en lo socioeconómico sino en reclamos vinculados al "mundo de la vida". También se dio al mismo tiempo un ingreso al espacio político y público de otros actores, por ejemplo los empresarios, con sus reclamos y demandas al Estado. 
En la investigación realizada entre 2003-20055, partiendo de inquietudes y demandas de los actores locales buscábamos conocer las transformaciones operadas en la organización y en la participación barrial en el marco de una matriz neoliberal y ante la situación de crisis. Uno de los elementos que surgió con fuerza desde las organizaciones locales fue la constatación de su transformación. De ser organizaciones de vecinos que se preocupaban por la mejora de las calles, el alumbrado, el transporte público, integrado básicamente por personas que vivían las mismas situaciones, trabajadores de fábricas ubicadas en la misma zona o cercanas, se transformaron en quienes atendían y sostenían a la población más vulnerable a través de merenderos, centros de primera infancia, centros juveniles. Para ello tuvieron que sacar personería jurídica, conveniar con el Estado departamental o nacional, contratar técnicos, administrar recursos. Una vez insertos en esta dinámica, sentían que no podían "volver atrás".

El reposicionamiento y recentramiento de políticas en el Estado, con mayor incidencia y regulación, plantea una desigualdad respecto a la sociedad civil que produce transformaciones en espacio, tiempo, modalidades organizativas y de participación, roles.

En el caso de Montevideo, la asunción del Frente Amplio de la Intenden- cia Departamental en 1990 buscó contraponer al modelo imperante a nivel internacional y nacional, una propuesta de descentralización y participación ciudadana. Para ello se dividió el territorio en dieciocho zonas y se generó una nueva institucionalidad (a través de juntas locales políticas y concejos vecinales, entre otros organismos). Se reforzó la idea de "vecindad", donde las personas que tradicionalmente se agrupaban en relación a sus barrios como lugares de pertenencia, identidad, cercanía, pasaron a ser parte de zonas no solo más amplias sino de mayor heterogeneidad, y a tener que intercambiar, pero también a priorizar, recursos siempre escasos (García y Neirotti, 1995). Esto sufre una nueva transformación cuando se vota la ley que crea los municipios. A su vez, las modalidades organizativas también se van transformando, no solo por la institucionalidad creada sino también por el fomento de las redes y la búsqueda de nuevas formas de participación, que ya se planteaba en crisis.

Cuando el Estado nacional retoma, desde 2005, un rol fuerte y desarrolla políticas públicas territorializadas, en Montevideo se asienta sobre las divisiones de zonas y municipios ya instaladas, pero instaura nuevas modalidades organizativas. Desde un modelo de redes, instala "mesas" que buscan ser una articulación interinstitucional e

5 Reconfiguración de espacios organizativos y participación ciudadana, ya citada. 
interactoral buscando romper (o mitigar) la fragmentación y superposición de políticas y acciones. Reunión tanto de técnicos como de vecinos y vecinas -de los pocos que participan activamente-, desplaza otras modalidades e instaura un "tiempo": en tanto son los técnicos quienes coordinan, se pasa del horario de la tarde/noche (luego del trabajo para los vecinos), al de las primeras horas de la tarde (horario de trabajo para los técnicos). Espacio y tiempo han ido redefiniéndose, así como los roles. Sin embargo, tanto desde la universidad como desde las políticas públicas, sigue haciéndose referencia a un actor y una organización que no necesariamente es la que hoy predomina, y se termina cayendo en el facilismo del "referente barrial", en torno a cuyas opiniones y disposiciones se elaboran análisis y propuestas. Por otro lado, la referencia a "sujetos colectivos" o a veces a movimientos y organizaciones, se reduce a un conjunto muy restringido que no necesariamente está definido por adscripción territorial. Se piensa además en adultos (no en niños o adolescentes), que se "reúnen" (a la manera tradicional). Una unidad en torno a valores, prioridades, sentidos comunes.

Las propuestas que buscan implementarse a partir de la Estrategia por la Vida y la Convivencia parten no solo de un "contrato" común, sino que suponen un otro con interés y capacidad de responder, por ejemplo, vecinos y vecinas de los barrios seleccionados. Preguntas simples sobre la sustentabi- lidad y sostenibilidad de las propuestas y procesos no son menores, ya que, por ejemplo, un equipamiento barrial que se rompe refuerza la sensación de no poder, la desvalorización de lo público y de las personas.

Ser ciudadano, el sentido de pertenencia, no tiene que ver solo con el Estado y el territorio, sino también con otras prácticas sociales y culturales. Cada participante se enfrenta a una situación social equipado con una biografía preestablecida de encuentros previos con los demás y con una gran gama de suposiciones culturales que cree compartidas. Se trata de prácticas situadas en un espacio y un tiempo, lógicas complejas, representaciones y creencias, sistemas de valores que ordenan, clasifican, jerarquizan, desde las cuales "leemos" y actuamos.

\section{Lo posible, lo pensable, lo deseable, lo problematizable}

La Estrategia por la Vida y la Convivencia propone la construcción de una alternativa social y cultural al "pensamiento y los valores dominantes y mercantilizados", buscando dar "cohesión" a la sociedad uruguaya, combinando convivencia y seguridad ciudadana. Desarrollar "cultura ciudadana",

conjunto de los comportamientos, valores, actitudes y percepciones que comparten los miembros de una sociedad y que determinan las formas y la calidad de la convivencia, influyen sobre el respeto del patrimonio común, y facilitan o dificultan el recono- 
cimiento de los derechos y los deberes ciudadanos (EVC, p. 7).

Los habitantes de una zona, de un barrio, ¿desde dónde miran el mundo y construyen su "realidad", desde dónde definen lo posible/imposible, lo pensable/no pensable? Las propuestas incluidas en las políticas públicas que buscan una "mejor" convivencia parecen basarse en la idea de que hay "una forma", "una realidad", y centrarse más en ideas de "adaptación" que de "construcción", "recreación" o "creación" (de un "juego" diferente). Se parte de una idea de lo "normal", lo "esperable", que se da por compartida.

Llegamos a ser humanos en una relación abierta al mundo, donde somos a la vez productores y producidos. La existencia se desarrolla empíricamente en un contexto de orden, dirección y estabilidad, que se expresa en relatos, en el lenguaje, en el cuerpo. "Alumbramos mundos" posibles. Los actos repetidos con frecuencia generan pautas que son aprehendidas, que pueden reproducirse con economía de esfuerzos, rutinas, conocimientos que se dan por establecidos y que sirven de base a proyectos, restringiendo las opciones, las decisiones, pero también abriendo a la deliberación y a la innovación (Berger y Luckmann, 2001). La cristalización de las instituciones hace que se experimenten como una realidad propia, que se presenta como hecho externo y coercitivo: "así se hacen las cosas" (hechos innegables), modos de "comportarse". El hombre ordinario presta al discurso el servicio de figurar en él como principio de totalización y como principio de acreditación: le permite decir "es cierto para todos" y "es la realidad de la historia" (Certeau, 2000, p. 53).

Las formaciones sociales se transmiten a las nuevas generaciones como tradición. Desde gobernantes, sociedad civil, medios, se habla del debilitamiento de las instituciones, partiendo de diferentes definiciones, niveles, horizontes de sentido. El lugar de la familia, de la educación, de lo local, recobra nueva centralidad en relación a la construcción colectiva. Dónde, cómo, entre quiénes se están construyendo consensos operativos, en relación a qué y hasta dónde. La redefinición de una situación involucra no solo al presente sino también al pasado.

Pretendemos aportar a mirar los procesos como más "multiformes", más ligados a la cotidianidad, poniendo en juego cuerpo, tiempo, espacio, acciones. Poner el énfasis no en atributos fijos sino en fuerzas y movimientos, los "saberes históricos de la lucha en la cotidianidad" (Foucault, 1979). La posibilidad de establecer "redes" de relaciones que busquen hacer más complejas la clasificación y jerarquización. Cómo se dan los condicionamientos, el control, la mirada del otro como normalizadora, la "infracción" (romper con lo normal) como algo que lesiona a la sociedad, los "castigos" se ponen en juego. ¿Cómo elegimos nuestros "territorios" de lucha? ¿Cómo entran las organizaciones de las que formamos parte y que nos forman? 
Trabajar en relación a encuentros, ocasiones, eventos, marcas, que permiten conocer lo que se valora, cómo y por qué, sin necesariamente tener que generar relatos o "historias" únicas.

Problematizar la idea del espacio público como aquel que permite la coexistencia pacífica y armoniosa, donde se confirma que podemos estar juntos, ser reconocidos (Delgado, abriljulio de 2011). Espacio ideal que las desigualdades, las diferencias, los estigmas, los miedos llevan a profundizar vulnerabilidades y vulneraciones.

¿Qué es lo que se ve como "deseable", como "correcto", como "deben ser las cosas" en un barrio, en relación a un espacio público? ¿Se comparte una idea común? ¿Qué se pone en juego en lo cotidiano? ¿Cómo y desde quiénes se define cuál es el "problema"? ¿Los conflictos? ¿Las transformaciones? Resolver problemas tiene que ver con la capacidad de imaginarlos.

En la ciudad se visibiliza la diversidad, marcas o "tachaduras", donde discursos, acciones y redes estructuran los relacionamientos. No son solo soporte, sino parte constitutiva de las personas. Construidos pero a la vez constructores de quienes los habitan, y a su vez crean los vínculos y los sentidos. Organizador de la diversidad, se dan allí choques, negociaciones, alianzas, enfrentamientos. La pertenencia territorial actúa como mediación para la constitución de identidades urbanas y para la movilización política. Es el lugar de comunicación de distintas "sociedades" entre sí. Se despliegan formas de vivir, de comunicarse, de exponerse y de replegarse. En territorios reales o simbólicos se expresan los mundos de vida, los grupos, las corporaciones, las "tribus", donde se dan luchas por la apropiación y definiciones legítimas de objetos y prácticas sociales, fuente de representaciones e imaginarios que orientan la acción. Hay una "escritura colectiva", donde nadie es solo escritor o lector, donde quienes transitamos participamos, lo queramos o no, lo sepamos o no.

La idea de convivir, vivir con la comunidad, el territorio y el rol de los actores sociales aparece como algo nombrado, dado por hecho, pero no necesariamente problematizado. Remite a cómo concebimos al otro, la otra, los otros. El filósofo italiano Esposito (2009) parte de la crítica hacia la idea de comunidad como "propiedad" de los sujetos, un atributo que hace que pertenezcan al mismo "conjunto", una "sustancia", algo que se agrega y los hace ser sujetos de una entidad "mayor" o mejor, una pertenencia que se puede perder y encontrar. Propone que comunidad refiere más a un deber o deuda, la obligación contraída con el otro, que lleva a la pregunta sobre ese otro qué es conmigo.

La ciudadanía y los derechos "hablan" de la lucha del reconocimiento de los otros como sujetos, con "intereses válidos, valores pertinentes y demandas legítimas". Buscamos trabajar en un marco de derechos reconceptua- 
lizados "como principios reguladores de las prácticas sociales, definiendo las reglas de las reciprocidades esperadas en la vida en sociedad a través de la atribución mutuamente acordada (y negociada) de las obligaciones y responsabilidades, garantías y prerrogativas de cada uno" (García Canclini, 1995 , p. 20). Supone tanto reivindicar los derechos de acceder y pertenecer al sistema sociopolítico como el derecho a participar en la reelaboración del sistema, definir por tanto aquello en lo cual queremos ser incluidos.

Romper con la intolerancia, la negación del otro, el miedo, la indiferencia. Recuperar la palabra de otros, de los procesos comunicativos, imbricados en la interacción cotidiana. "Una de las formas de exclusión ciudadana se da en la desposesión del derecho a ser visto y oído" (Martín Barbero, 2001, p. 44). Lo que debe buscar una comunicación democrática es posibilitar visibilidades, "existencias", posibilidades de "contar" socialmente, tanto individuales como colectivas.

Como comunicadores podemos profundizar en cómo se construyen sentidos. Desde la multiplicidad de "lugares", medios, espacios. Comunicación que no solo son palabras, son gestos, actitudes, formas de tratarse. Se da cotidianamente, en la posibilidad de construir una visión desde la experiencia personal, desde los códigos propios. Propender a articular lo micro y lo macro, lo cotidiano y lo eventual, lo lúdico y lo "serio". Traba- jar en modalidades que permitan distintas formas de entrar, salir, estar, en la escena pública. Donde cada uno se sienta respetado, escuchado, atendido, entendido, legitimando los discursos y las formas de representarse de cada persona o grupo. La construcción de la democracia, de la participación, de la diversidad, se da en los espacios de interacción pero también en sus bordes o fronteras.

\section{Referencias}

Bateson, G. (1997). Espíritu y naturaleza. Buenos Aires: Amorrortu Editores.

Berger, P., y Luckmann, T. (2001). La construcción social de la realidad. Buenos Aires: Amorrortu Editores.

Bourdieu, P. (2002). Campo de poder, campo intelectual. Buenos Aires: Montressor.

Castoriadis, C. (1989). La institución imaginaria de la sociedad. Vol. 2: El imaginario social y la institución. Barcelona: Tusquets Editores.

Causa Abierta Portal de Noticias (25 de abril de 2013). Olesker afirmó que Uruguay es el país de menor desigualdad en Latinoamérica. Recuperado de http://www.causaabierta.com.uy/olesker-afirmoque-uruguay-es-el-pais-de-menordesigualdad-de-latinoamerica/ Certeau, M. de (2000). La invención de lo cotidiano, I. Artes de hacer. México: Instituto Tecnológico y de Estudios Superiores de OccidenteUniversidad Iberoamericana. 
Coraggio, J. L. (2007). Economía social, acción pública y política. Buenos Aires: Ciccus.

Delgado, M. (abril-junio de 2011). El espacio público no existe. Barcelona Metrópolis. Recuperado de http:// w2.bcn.cat/bcnmetropolis/arxiu/es/ pageeb68.html?id=21\&ui=507

Esposito, R. (2009). Communitas, nada en común. Recuperado de http://accesoamateriales.blogspot.com/2009/06/ communitas-roberto-esposito.html

Foucault, M. (1979). Microfísica del poder. Madrid: Ediciones La Piqueta.

García, A., y Neirotti, E. (1995). Con ojos de educador popular. Una mirada al proceso de descentralización municipal en Montevideo. En Construcción de experiencias. Bogotá: CEAAL.

García Canclini, N. (1995). Consumidores y ciudadanos. Conflictos multiculturales de la globalización. México: Grijalbo.

Leal, G. (s. f.). Más barrio, más vida (documento). Montevideo.

Martín Barbero, J. (2001). Televisión pública, televisión cultural: entre la renovación y la invención. En O. Rincón (Comp.), Televisión pública: del consumidor al ciudadano. Bogotá: Convenio Andrés Bello.

Morin, E. (1999). El método: la naturaleza de la naturaleza. Madrid: Cátedra.

Paternain, R. (2 de febrero de 2011). Hay una nueva hegemonía conservadora en el abordaje de la inseguridad". Vadenuevo Mensual, 29. Año 3. Entrevista a Rafael Paternain, realizada por Pedro Cribari, Federico Martínez y Miguel Muyala. Montevideo. Recuperado de www.vadenuevo.com.uy

Presidencia de la República Oriental del Uruguay, Gabinete de Seguridad. (26 junio de 2012). Estrategia para la Vida y la Convivencia (EVC). Medidas que promueven la convivencia social. Documento. Recuperado de http://www.presidencia.gub.uy/ Comunicacion/comunicacionNoticias/gabinete-seguridad-presentopaquete-15-medidas-promoverconvivencia-ciudadana

Reguillo, R. (marzo de 1997). Ciudad y comunicación: densidades, ejes y niveles. Diálogos de la Comunicación, 47.

Rosanvallon, P. (2012). La sociedad de iguales. Buenos Aires: Manantial. 